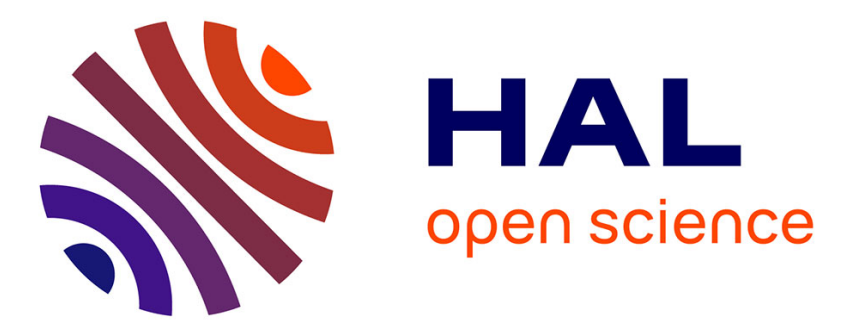

\title{
Modélisation des problèmes d'impact avec dissipation d'énergie par frottement
}

Benoit Magnain, Zhi-Qiang Feng, Jean-Michel Cros

\section{To cite this version:}

Benoit Magnain, Zhi-Qiang Feng, Jean-Michel Cros. Modélisation des problèmes d'impact avec dissipation d'énergie par frottement. Comptes Rendus Mécanique, 2005, 333 (5), pp.419-424. 10.1016/j.crme.2005.03.002 . hal-01179488

\section{HAL Id: hal-01179488 \\ https://hal.science/hal-01179488}

Submitted on 28 Jun 2018

HAL is a multi-disciplinary open access archive for the deposit and dissemination of scientific research documents, whether they are published or not. The documents may come from teaching and research institutions in France or abroad, or from public or private research centers.
L'archive ouverte pluridisciplinaire HAL, est destinée au dépôt et à la diffusion de documents scientifiques de niveau recherche, publiés ou non, émanant des établissements d'enseignement et de recherche français ou étrangers, des laboratoires publics ou privés. 


\title{
Modélisation des problèmes d'impact avec dissipation d'énergie par frottement
}

\author{
Benoît Magnain, Zhi-Qiang Feng *, Jean-Michel Cros \\ Laboratoire de mécanique et d'énergétique d'Évry, université d'Évry - Val d'Essonne, 40, rue du Pelvoux, 91020 Évry cedex, France
}

\section{Résumé}

La méthode du bi-potentiel a été appliquée avec succès au traitement en statique des problèmes de contact avec frottement. Cet article présente l'extension de cette méthode à l'analyse des problèmes de contact en dynamique et en présence de plusieurs corps déformables. Dans ce but, un algorithme du premier ordre est utilisé pour la discrétisation temporelle des équations du mouvement. Les résultats numériques obtenus montrent clairement la dissipation d'énergie introduite par le frottement entre les différents solides en contact.

\begin{abstract}
Modeling of impact problems with energy dissipation by friction. The bi-potential method has been successfully applied for the modeling of frictional contact problems in static cases. This paper presents the application of this method for dynamic analysis of impact problems with multiple deformable bodies. A first order algorithm is applied for numerical integration of the time-discretized equation of motion. The numerical results show clearly the physical energy dissipation introduced by frictional effects between the solids in contact.
\end{abstract}

Mots-clés : Mécanique des solides numérique ; Impact ; Dissipation d'énergie

Keywords: Computational solid mechanics; Impact; Energy dissipation

\footnotetext{
* Corresponding author.

Adresses e-mail : magnain@iup.univ-evry.fr (B. Magnain), feng@iup.univ-evry.fr (Z.-Q. Feng), cros@iup.univ-evry.fr (J.-M. Cros).
} 


\section{Abridged English version}

Problems involving contact and friction are among the most difficult ones in mechanics and at the same time of crucial practical importance in many engineering branches. The main mathematical difficulty lies in the severe contact non-linearities. In the past decade, substantial progress has been made in the analysis of contact problems using finite element procedures. A large number of algorithms for the numerical solution of the related finite element equations and inequalities have been presented in the literature. De Saxcé and Feng [1,2] proposed a theory called ISM (Implicit Standard Materials) and a bi-potential method, in which an augmented Lagrangian formulation was developed. The aim of the present paper is to apply the bi-potential method for contact modeling in dynamic cases in the field of Non-Smooth Dynamics using a first order algorithm for integration of the equation of motion. The proposed method is applied to simulate the impact of two elastic bars and the impact of two elastic cylinders in rigid walls. In order to show the physical energy dissipation by frictional effects, frictionless and frictional contact are considered for the second example. After finite element discretization, non-linear dynamic mechanical behaviors of solid media with contact are governed by (1). For integrating this dynamics equation, Jean $[6,7]$ has proposed a first order algorithm which is used in this work. Using the linearization of the force vector (2), we obtain a recursive form of (1) in terms of displacements (3). At the end of each time step, the velocity is updated by (4). Feng [8] has proposed a solution strategy which consists in separating the nonlinearities of (3). First, the vector $\mathbf{R}_{c}$ is determined by the bi-potential method in a reduced system, which only concerns contact nodes. Then, the vector $\Delta \mathbf{u}$ can be computed in the whole structure, using contact reactions as external loading. The classical impact of two elastic bars [10] is performed so as to validate the proposed approach (Figs. 1-4). The second example concerns the impact of two cylinders inside rigid walls (proposed by Armero and Petocz [3]). The problem is displayed in Fig. 5. Figs. 6 and 7 show the plots of the kinetic energy $E_{\mathrm{c}}$, the elastic strain energy $E_{\mathrm{e}}$ and the total energy $\left(E_{\mathrm{t}}=E_{\mathrm{c}}+E_{\mathrm{e}}\right)$. We can observe clearly that the total energy is perfectly conserved in the case of frictionless contact (Fig. 6). However, in the case of frictional contact $(\mu=0.2)$, the total energy decreases at each shock (Fig. 7). Thus the energy is dissipated by frictional effects as expected. It is interesting to note from Fig. 6 that the left cylinder hits another one at $t=2.45 \mathrm{~s}$, without friction, whereas this changes to $t=2.8 \mathrm{~s}$ in the case of frictional contact as indicated in Fig. 7. This fact can be explained as follows: because of friction forces, the rebounding direction is changed such that the running distance of the left cylinder from the bottom wall to the right cylinder becomes longer. Thus, it takes more time to hit each other.

\section{Introduction}

Dans de nombreux domaines, les problèmes de contact avec frottement jouent un rôle considérable. Toutefois, leur résolution comporte de nombreuses difficultés dont la première est la non-linéarité des lois qui les définissent. Au cours de la dernière décennie, de larges progrès ont été réalisés dans l'analyse de ce type de problèmes à l'aide de la méthode des éléments finis et un grand nombre d'algorithmes de résolution numérique a été présenté dans la littérature. Parmi les plus utilisés, citons la méthode de pénalisation et la méthode des multiplicateurs de Lagrange. De Saxcé et Feng [1,2] ont proposé une théorie appelée MSI (Matériau Standard Implicite) et une méthode du bi-potentiel dans laquelle une nouvelle formulation du Lagrangien augmenté est développée. Cette dernière est très différente des autres, en particulier dans le fait que le problème du contact est traité dans un système réduit par un algorithme très efficace de type prédiction-correction. De plus, elle conduit à un unique problème variationnel en déplacement et une seule inégalité. Ainsi, le contact unilatéral et le frottement sont couplés via un bi-potentiel de contact. Par ailleurs, l'analyse de la dynamique des structures par des méthodes implicites se fait le plus souvent à l'aide de schémas d'intégration du second ordre comme ceux de Newmark, Wilson, etc. Cependant, il est bien connu que dans les problèmes d'impact, les champs de vitesse et d'accélération ne sont pas continus au cours du temps à cause des changements brusques des conditions de contact. L'utilisation de ce type d'algorithme peut donc mener à de sérieuses erreurs dans les résultats. Différents auteurs, [3,4] par exemple, proposent des méthodes pour 
corriger ces problèmes, via par exemple des techniques de régularisation mais qui entraînent des développements complexes. Une autre alternative, pour palier ces difficultés, est l'utilisation d'algorithmes du premier ordre [5-7]. L'intérêt de cette approche réside à la fois dans sa simplicité mais également dans son efficacité. Le propos de cet article est d'appliquer la méthode du bi-potentiel à la modélisation du contact en dynamique dans le cadre des grands déplacements en utilisant un algorithme du premier ordre. Un premier exemple, dont on connaît la solution analytique, permet de valider la combinaison d'algorithmes dans le cas sans frottement. Un second exemple, impact de deux cylindres élastiques enfermés entre quatre murs rigides, permet de montrer la dissipation d'énergie par frottement. Pour ce dernier, le cas sans frottement et celui avec frottement est considéré.

\section{Méthode du bi-potentiel et schéma d'intégration du premier ordre}

La résolution d'un problème de dynamique consiste à prendre en compte, par rapport au cas sans contact, les réactions au niveau des surfaces de contact. Pour déterminer ces réactions, la méthode du bi-potentiel est utilisée. Cette dernière a été abondamment décrite, tant au niveau continu que discret $[1,2,8]$ avec de nombreux exemples numériques, qui démontrent son efficacité pour traiter les problèmes de contact unilatéral avec modèle de frottement de Coulomb. Notamment, elle comprend une manière élégante de traiter les potentiels non différentiables qui apparaissent en mécanique non linéaire. Ainsi, après discrétisation spatiale par la méthode des éléments finis (approche Lagrangienne totale), un problème de contact en dynamique et dans le cas non-linéaire s'écrit sous la forme de l'équation d'équilibre suivante :

$$
\mathbf{M u ̈}=\mathbf{F}+\mathbf{R}_{\mathrm{c}}, \quad \text { où } \mathbf{F}=\mathbf{F}_{\mathrm{ext}}+\mathbf{F}_{\text {int }}-\mathbf{C} \dot{\mathbf{u}}
$$

Les vecteurs $\mathbf{F}_{\text {ext }}, \mathbf{F}_{\text {int }}$ et $\mathbf{R}_{\mathrm{c}}$ représentent respectivement les forces externes, internes, et de contact. $\mathbf{M}$ est la matrice de masse du système et $\mathbf{C}$ la matrice d'amortissement. Le vecteur $\mathbf{R}_{c}$ est déterminé en premier par la méthode du bi-potentiel. Puis, le vecteur $\Delta \mathbf{u}$ est calculé en considérant les réactions de contact comme un chargement externe. Il est très important de noter que, par opposition à la méthode de pénalisation ou celle des multiplicateurs de Lagrange, la méthode du bi-potentiel ne modifie jamais la structure de la matrice globale de rigidité, ni n'augmente le nombre de degrés de liberté. Une conséquence intéressante de cette propriété est qu'il est facile d'implanter cette méthode dans un code éléments finis classique afin de traiter les problèmes de contact avec frottement. L'intégration de l'Éq. (1) est réalisée par un schéma du premier ordre [6,7]. La dynamique est dans ce cas écrite au sens des mesures différentielles. Les équations ne font alors plus intervenir l'accélération qui pose des difficultés numériques au moment de l'impact. La linéarisation du vecteur force donne :

$$
\mathbf{F}^{i+1}=\mathbf{F}_{\mathrm{int}}^{i}+\frac{\partial \mathbf{F}}{\partial \mathbf{u}}\left(\mathbf{u}^{i+1}-\mathbf{u}^{i}\right)+\frac{\partial \mathbf{F}}{\partial \dot{\mathbf{u}}}\left(\dot{\mathbf{u}}^{i+1}-\dot{\mathbf{u}}^{i}\right)=\mathbf{F}_{\mathrm{int}}^{i}-\mathbf{K}^{i} \Delta \mathbf{u}-\mathbf{C}^{i} \Delta \dot{\mathbf{u}}
$$

Finalement, nous obtenons la forme récursive en déplacement suivante $(0 \leqslant \xi \leqslant 1$ et $0 \leqslant \theta \leqslant 1)$ :

$$
\left\{\begin{array} { l } 
{ \overline { \mathbf { K } } ^ { i } \Delta \mathbf { u } = \overline { \mathbf { F } } ^ { i } + \overline { \mathbf { F } } _ { \mathrm { acc } } ^ { i } + \mathbf { R } _ { \mathrm { c } } ^ { i + 1 } } \\
{ \mathbf { u } ^ { i + 1 } = \mathbf { u } ^ { i } + \Delta \mathbf { u } }
\end{array} \quad \text { avec } \left\{\begin{array}{l}
\overline{\mathbf{K}}^{i}=\xi \mathbf{K}^{i}+\frac{\xi}{\theta \Delta t} \mathbf{C}^{i}+\frac{1}{\theta \Delta t^{2}} \mathbf{M}^{i} \\
\overline{\mathbf{F}}_{\mathrm{acc}}^{i}=-\frac{1}{\theta \Delta t^{2}} \mathbf{M}^{i}\left\{\mathbf{u}^{i}-\mathbf{u}^{t}-\Delta t \dot{\mathbf{u}}^{t}\right\} \\
\overline{\mathbf{F}}^{i}=(1-\xi)\left(\mathbf{F}_{\mathrm{int}}^{t}+\mathbf{F}_{\mathrm{ext}}^{t}\right)+\xi\left(\mathbf{F}_{\mathrm{int}}^{i}+\mathbf{F}_{\mathrm{ext}}^{t+\Delta t}\right)
\end{array}\right.\right.
$$

À la fin de chaque pas de temps, la vitesse est réactualisée par :

$$
\dot{\mathbf{u}}^{t+\Delta t}=\left(1-\frac{1}{\theta}\right) \dot{\mathbf{u}}^{t}+\frac{1}{\theta \Delta t}\left(\mathbf{u}^{t+\Delta t}-\mathbf{u}^{t}\right)
$$

On cherche, pour le cas d'impact sans frottement, à vérifier le principe de conservation de l'énergie et à quantifier l'énergie dissipée lors des impacts avec frottement. Pour cela, il est possible d'agir sur les paramètres du schéma d'intégration. Pour $\theta=0,5$ ce schéma correspond à la règle du point milieu implicite. Simo et Wong [9] ont montré 


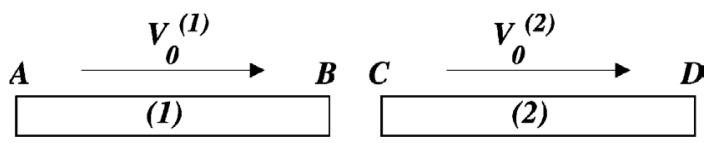

Fig. 1. Impact de deux barres élastiques.

Fig. 1. Longitudinal impact of two elastic bars.

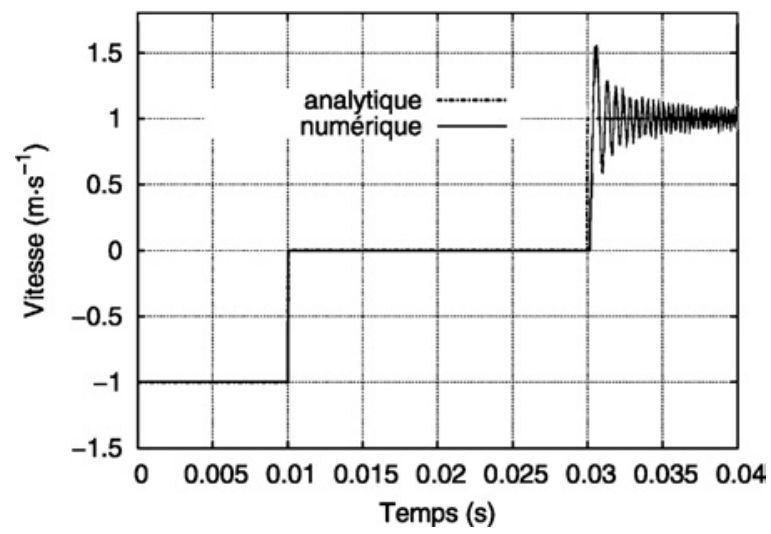

Fig. 3. Vitesse du point C.

Fig. 3. Local velocity of point C.

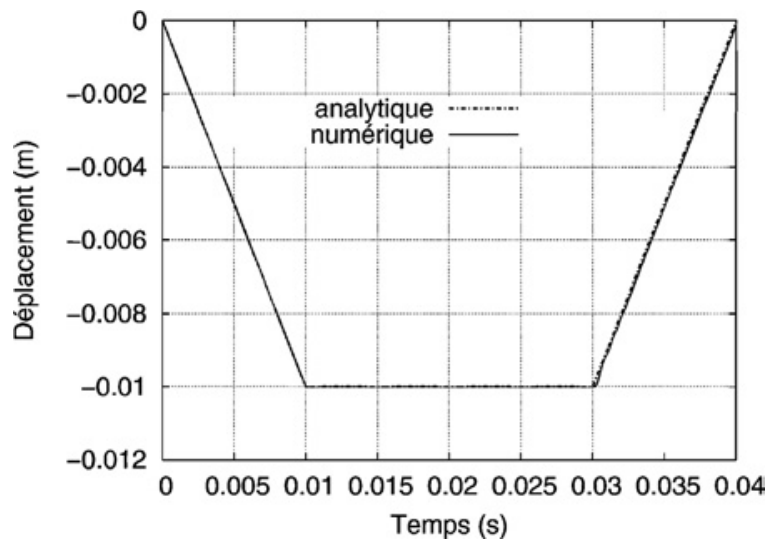

Fig. 2. Déplacement du point $\mathrm{C}$.

Fig. 2. Local displacement of point $\mathrm{C}$.

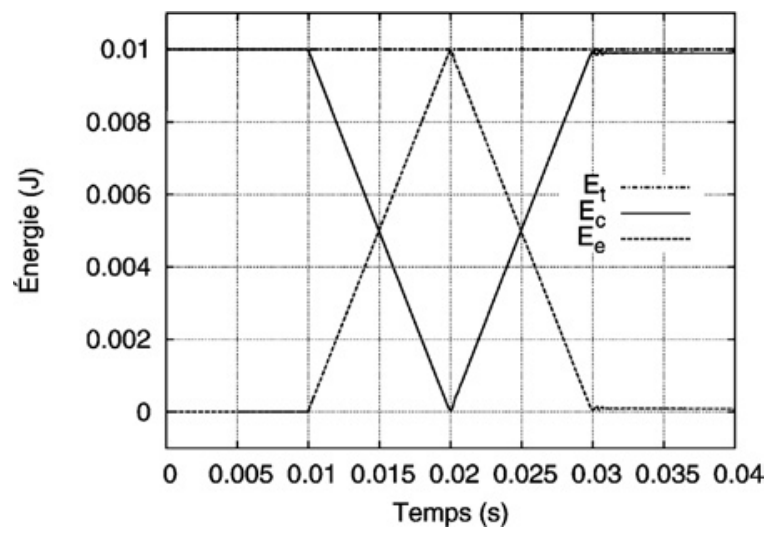

Fig. 4. Évolution de l'énergie.

Fig. 4. Energy evolution.

que ce schéma préserve l'énergie totale et l'équilibre pour les problèmes dynamiques sans contact. Dans la suite, on prendra $\theta=\xi=0,5$. L'expérience montre que pour ces valeurs, le schéma d'intégration est stable et qu'il préserve l'énergie totale dans le cas sans frottement. Les exemples numériques vont montrer que ce choix permet également d'assurer la stabilité pour les problèmes d'impact avec frottement. Pour des valeurs de $\xi$ et de $\theta$ plus grandes, on constate l'apparition d'une dissipation numérique.

\section{Résultats numériques}

On traite, dans un premier temps, l'exemple classique [10] de l'impact de deux barres élastiques identiques (Fig. 1) de longueur $10 \mathrm{~m}$. Les caractéristiques du matériau sont $\rho=10^{-3} \mathrm{~kg} \mathrm{~m}^{-3}$ et $E=10^{3} \mathrm{~Pa}$. La vitesse initiale de chaque barre est respectivement $v_{0}^{(1)}=1 \mathrm{~m} \mathrm{~s}^{-1}$ et $v_{0}^{(2)}=-1 \mathrm{~m} \mathrm{~s}^{-1}$. La simulation dure $0,04 \mathrm{~s}$ pour un pas de temps égal à $10^{-5}$ s. Les Figs. 2 et 3 représentent respectivement le déplacement et la vitesse du point $\mathrm{C}$. Les oscillations de la solution numérique (Fig. 3) traduisent les vibrations de la barre suite à l'impact phénomène 


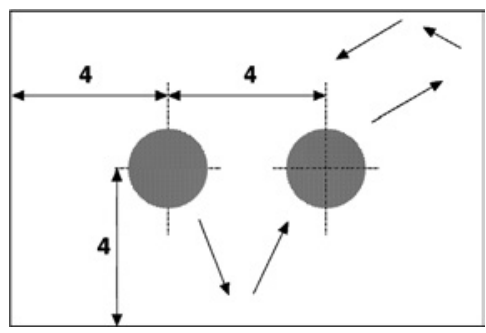

Fig. 5. Impact entre deux cylindres.

Fig. 5. Impact between two cylinders.

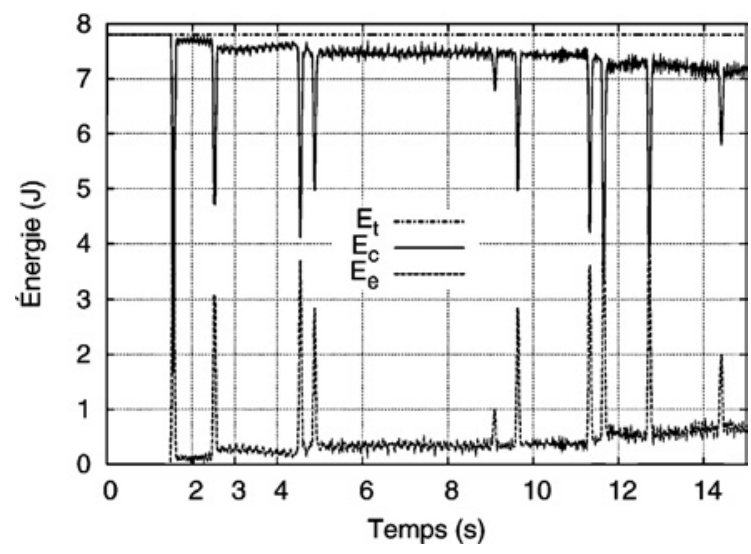

Fig. 6. Évolution de l'énergie : sans frottement.

Fig. 6. Energy evolution: without friction.

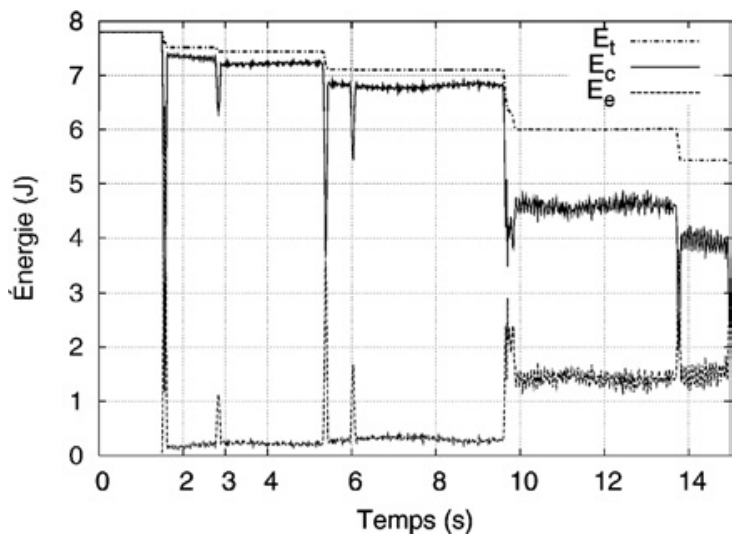

Fig. 7. Évolution de l'énergie : avec frottement.

Fig. 7. Energy evolution: with friction.

que ne peut représenter le modèle analytique. Ces oscillations indiquent la présence d'une énergie de déformation élastique (même faible) dans la barre.

L'évolution de l'énergie du système constitué par les deux barres est tracée sur la Fig. 4 , où $E_{\mathrm{c}}, E_{\mathrm{e}}$ et $E_{\mathrm{t}}=$ $E_{\mathrm{c}}+E_{\mathrm{e}}$ sont respectivement l'énergie cinétique, l'énergie de déformation et l'énergie totale. Ces résultats montrent de manière probante le bon comportement de la méthode proposée qui permet de vérifier le principe de conservation de l'énergie dans le cas d'un impact sans frottement.

L'exemple suivant simule l'impact entre deux cylindres enfermés entre quatre murs rigides (exemple initialement étudié par Armero et Petocz [3]). Nous souhaitons ainsi tester les performances de notre méthode sur un problème présentant un enchaînement complexe de séquences de contact. Le diamètre initial des cylindres est égal à $2 \mathrm{~m}$. Nous considérons que le matériau constituant les deux cylindres vérifie le modèle de Saint-Venant-Kirchhoff avec les paramètres suivant : $E=2700 \mathrm{~Pa}, \rho=1 \mathrm{~kg} \mathrm{~m}^{-3}$ et $v=0,33$. Le cylindre de gauche se voit imposer une vitesse initiale $\left(v_{x}=1 \mathrm{~m} \mathrm{~s}^{-1}\right.$ et $\left.v_{y}=-2 \mathrm{~m} \mathrm{~s}^{-1}\right)$ de telle sorte qu'il vient impacter le mur du bas puis, après un rebond, le cylindre de droite (Fig. 5).

La simulation s'étend sur une durée totale de $15 \mathrm{~s}$ avec un pas de temps $\Delta t=10^{-3} \mathrm{~s}$. La Fig. 6 montre l'évolution de l'énergie cinétique $E_{\mathrm{c}}$, de l'énergie potentielle élastique $E_{\mathrm{e}}$ ainsi que celle de l'énergie totale $\left(E_{\mathrm{t}}=E_{\mathrm{c}}+E_{\mathrm{e}}\right)$ dans le cas où le contact est considéré sans frottement $(\mu=0)$. Nous y observons très clairement la conservation de l'énergie totale tout au long du mouvement malgré l'évolution complexe de $E_{\mathrm{c}}$ et $E_{\mathrm{e}}$. Au contraire (Fig. 7), dans le cas où le contact se fait avec frottement (ici $\mu=0,2$ ), il y a dissipation de l'énergie totale durant chaque choc. Ainsi, l'énergie totale est dissipée par l'effet du frottement, comme prévu. Il est intéressant de noter sur la Fig. 6 que l'impact entre les deux cylindres se produit à l'instant $t=2,45 \mathrm{~s}$ dans le cas sans frottement alors qu'il ne 
survient qu'à $t=2,8 \mathrm{~s}$ (Fig. 7) s'il y a du frottement. Cela peut s'expliquer par le fait que les forces de frottement changent légèrement la direction suivant laquelle le premier cylindre rebondit contre le mur : la distance entre les futurs points de contact, qui ne sont plus les mêmes, est alors plus longue. De même, le choc est moins important puisqu'il n'est plus parfaitement frontal, ce qui se traduit par une production d'énergie de déformation élastique moins importante lors de l'impact entre les deux cylindres.

\section{Conclusion}

Dans cette Note, la modélisation de problèmes d'impact avec frottement est traitée à l'aide de la méthode du bipotentiel associée à un schéma d'intégration du premier ordre. Les résultats numériques présentés démontrent que l'algorithme proposé possède de grandes qualités tant en terme de précision qu'en terme de stabilité. De plus, un des exemples permet de conclure clairement à une bonne prise en compte des effets du frottement sur la dissipation d'énergie totale.

\section{Références}

[1] G. De Saxcé, Z.-Q. Feng, New inequality and functional for contact with friction: the implicit standard material approach, Mech. Struct. \& Mach. 19 (1991) 301-325.

[2] G. De Saxcé, Z.-Q. Feng, The bi-potential method: a constructive approach to design the complete contact law with friction and improved numerical algorithms, in: Recent Advances in Contact Mechanics, special issue, Math. Comp. Modeling 28 (4-8) (1998) $225-245$.

[3] F. Armero, E. Petocz, Formulation and analysis of conserving algorithms for frictionless dynamic contact/impact problems, Comput. Methods Appl. Mech. Engrg. 158 (1998) 269-300.

[4] T.A. Laursen, G.R. Love, Improved implicit integrators for transient impact problems geometric admissibility within the conserving framework, Int. J. Numer. Methods Engrg. 53 (2002) 245-274.

[5] O.C. Zienkiewicz, W.L. Wood, R.L. Taylor, An alternative single-step algorithm for dynamic problems, Earthg. Engrg. Struct. Dyn. 8 (1980) 31-40.

[6] M. Jean, Dynamics with partially elastic shocks and dry friction: double scale method and numerical approach, in: Proc. 4d Meeting on Unilateral Problems in Structural Analysis, Capri, 1989.

[7] M. Jean, The non-smooth contact dynamics method, Comput. Methods Appl. Mech. Engrg. 177 (1999) 235-257.

[8] Z.-Q. Feng, 2D or 3D frictional contact algorithms and applications in a large deformation context, Comm. Numer. Methods Engrg. 11 (1995) 409-416.

[9] J.C. Simo, K.K. Wong, Unconditionally stable algorithms for rigid body dynamics that exactly preserve energy and momentum, Int. J. Numer. Methods Engrg. 31 (1991) 19-52.

[10] N. Hu, A solution method for dynamic contact problems, Computers \& Structures 63 (1997) 1053-1063. 